\title{
Determination of Fatty Acids and Carbohydrate Monomers in Micro-organisms by means of Glass Capillary Gas Chromatography: Analysis of Mycobacterium gordonae and Mycobacterium scrofulaceum
}

\author{
By CHRISTER ALVIN, ${ }^{1}$ LENNART LARSSON,${ }^{2 *}$ \\ MOGENS MAGNUSSON, ${ }^{3}$ PER-ANDERS MÅRDH, ${ }^{2}$ GÖRAN ODHAM, ${ }^{4}$ \\ AND GUNILLA WESTERDAHL ${ }^{4}$ \\ ${ }^{1}$ Department of Technical Analytical Chemistry, University of Lund, Sweden \\ ${ }^{2}$ Department of Medical Microbiology, University of Lund, Sölvegatan 23, S-223 62 Lund, \\ Sweden \\ ${ }^{3}$ Tuberculin Department, Statens Seruminstitut, Copenhagen, Denmark \\ ${ }^{4}$ Laboratory of Ecological Chemistry, University of Lund, Sweden
}

(Received 12 July 1982)

\begin{abstract}
Trifluoroacetylated whole-cell methanolysates of four strains each of Mycobacterium gordonae and Mycobacterium scrofulaceum were analysed by gas chromatography, using a glass capillary column. The major chromatographic peaks were identified by mass spectrometry as derivatives of fatty acids and carbohydrates. In addition, two predominant peaks, present in chromatograms representing $\boldsymbol{M}$. scrofulaceum, were identified as 2-octadecanol and 2-eicosanol. These secondary alcohols were not found in any of the strains of $M$. gordonae studied. The amount of tuberculostearic acid in the latter species was less than $1 \%$ of that in $M$. scrofulaceum.
\end{abstract}

\section{INTRODUCTION}

In recent years, a number of gas chromatographic (GC) methods for the characterization and identification of micro-organisms have been elaborated. Profiling of derivatives of cellular constituents such as fatty acids, carbohydrates and amino acids from pure cultures has been employed. Furthermore, extracellular microbial metabolites, particularly of anaerobic bacteria, have been studied by GC. The resulting chromatographic patterns are usually specific as to genus or sometimes as to species (Larsson \& Mårdh, 1977).

One approach to the GC study of microbial constituents includes mild fragmentation of lyophilized organisms with methanolic hydrogen chloride, followed by trimethylsilylation or trifluoroacetylation. In this way, free and bound fatty acids are converted to corresponding methyl esters, and carbohydrates, including polysaccharides, to silylated or trifluoroacetylated monomeric methyl glycosides. Provided that the correct GC conditions are chosen, these two classes of compounds can be detected in one and the same GC analysis (Jantzen et al., 1972).

The development of diagnostic techniques for the rapid identification of mycobacteria ought to receive high priority, since the methods used today are both laborious and time consuming. GC analysis of cellular fatty acids as used, e.g. by Tisdall et al. (1979) and Guerrant et al. (1981), made it possible to distinguish various mycobacterial species encountered in human infections. Larsson \& Mårdh (1976) and Larsson et al. (1979 a) employed both analysis of fatty acids and of trifluoroacetylated whole-cell methanolysates. In these latter studies, no attempts (other than those based on GC retention data) were made to establish the identities of the chromatographed compounds.

The present investigation aimed to optimize the chromatographic conditions for the simultaneous GC registration of both microbial carbohydrates and fatty acids, analysed as trifluoroacetyl (TFA) derivatized methyl glycosides and methyl esters, respectively. A glass 
capillary column was used in order to obtain the best possible resolution. Two scotochromogenic mycobacterial species were selected as test organisms, viz. Mycobacterium gordonae and $M$. scrofulaceum. Mass spectrometry (MS) was employed to establish the identity of the chromatographic peaks.

\section{METHODS}

Organisms. The mycobacteria studied comprised $M$. gordonae (MNC 1226, MNC 1227, MNC 1230 and MNC 1233) and $M$. scrofulaceum (MNC 1225 and MNC 1232), isolated from clinical specimens at the Department of Bacteriology, University Hospital, Lund, Sweden. Furthermore, two strains of $\boldsymbol{M}$. scrofulaceum (MNC 96 and MNC 98, obtained from Dr A. M. Masson, Montreal, Canada) were tested. All strains were identified by biochemical and immunological methods.

Each strain had been cultured on two slants of Löwenstein-Jensen medium. Bacteria were collected from each slant and inoculated into Proskauer-Beck medium (Difco) at $37^{\circ} \mathrm{C}$ for $28 \mathrm{~d}$. The organisms were autoclaved, washed, lyophilized and stored in a vacuum desiccator at reduced pressure until use (Larsson \& Mårdh, 1976).

Chemicals. All chemicals used were of analytical grade and used without further purification. Solvents were purchased from Merck and trifluoroacetic anhydride from Fluka (Buchs, Switzerland). Methyl tuberculostearate, used as a reference, was synthesized (Odham et al., 1979).

Derivatization. Lyophilized cells (4-6 mg) were stored overnight in an excess (about $1 \mathrm{ml}$ ) of $1 \mathrm{M}$-methanolic $\mathrm{HCl}$ in sealed glass ampoules at $80^{\circ} \mathrm{C}$ as previously described (Larsson et al., 1979a). After centrifugation of the samples the supernatants were transferred to $2 \mathrm{ml}$ glass tubes and evaporated to dryness at room temperature under a stream of dry nitrogen. Ethyl acetate $(20 \mu \mathrm{l})$ and trifluoroacetic anhydride $(30 \mu \mathrm{l})$ was added and the tubes sealed with Teflon-lined screw caps before being heated at $80^{\circ} \mathrm{C}$ for $10 \mathrm{~min}$. Then, after 10 to $15 \mathrm{~min}$ at room temperature, the samples were used directly for $\mathrm{GC}$ analysis.

Gas chromatography. A Varian, model 3700 , gas chromatograph equipped with a $25 \mathrm{~m}$ wall-coated glass capillary column (i.d. $0.2 \mathrm{~mm}, \mathrm{OV}-101$ ), and connected to a Varian model CDS-101 electronic integrator was used. A $0.1 \mathrm{~m}$ glass pre-column (i.d. $3.5 \mathrm{~mm}$ ) packed with $2 \% \mathrm{OV}-1$ on Chromosorb G, 45-60 mesh, was placed in the injector block and attached to the capillary column. The packing material in the pre-column was regularly renewed to remove non-volatile, carbonized compounds. An all-glass split device was inserted after the precolumn. The temperature of the injector was $220^{\circ} \mathrm{C}$ and that of the flame ionization detector $240^{\circ} \mathrm{C}$. The column temperature was programmed from 80 to $230^{\circ} \mathrm{C}$, at a rate of $5^{\circ} \mathrm{C} \mathrm{min}^{-1}$, and held at $230^{\circ} \mathrm{C}$ for $10 \mathrm{~min}$. The carrier gas (nitrogen) flow rate was $1.0 \mathrm{ml} \mathrm{min}^{-1}$ through the column; the split ratio was 1:50. Make-up gas (nitrogen) was introduced before the detector at a flow rate of $25 \mathrm{ml} \mathrm{min}^{-1}$.

Mass spectrometry. A Varian model MAT $112 \mathrm{GC} / \mathrm{MS}$ combination, was used. The ion source temperature was held at $240^{\circ} \mathrm{C}$ and the electron energy was $70 \mathrm{eV}$. The chromatographic conditions were those described above. The identities of the major peaks in the chromatograms were established both by the recording of mass spectra and by comparative studies on GC retention data of reference compounds. In addition, selected ion monitoring (SIM), focusing at $\mathrm{m} / \mathrm{z} 312\left(\mathrm{M}^{+}\right)$, was used in studies on $M$. gordonae for the demonstration of the methyl ester of tuberculostearic acid as previously described (Larsson et al., 1979b, 1980).

\section{RESULTS}

\section{Identity of peaks}

The chemical structure of the components corresponding to peaks 1 to 11 (Fig. 1) were deduced by GC and MS. The mass spectra of $(1-4)$ proved identical with those of derivatized authentic arabinose, galactose, mannose, and glucose, respectively, whereas $(5,6,8,9$ and 11$)$ proved consistent with the methyl esters of palmitoleic, palmitic, oleic, stearic and tuberculostearic acids. When analysing a methanolysate of $M$. scrofulaceum which had not been subjected to trifluoroacetylation, it was observed that the compounds representing peaks (7) and (10) eluted approximately 2 min later, than when such derivatization was made, indicating that they contained hydroxyl groups. The mass spectra of these two underivatized components showed neither a molecular peak, nor peaks characteristic of fatty acid methyl esters. Peaks in the high mass range of component 10 were found at $m / z 280, m / z 281$ and $m / z 283$, while the base peak was found at $m / z 45$. It is known that long-chain aliphatic alcohols give spectra where the molecular ion peaks are exceedingly small, even absent, and with characteristic mass fragments at $m / z \mathrm{M}-18$ and $m / z \mathrm{M}-15$. The spectrum therefore appeared consistent with a $\mathrm{C}_{20}$ saturated alcohol. Alpha-cleavage with respect to the carbon atom carrying the hydroxyl group, yielding the base peak at $m / z 45\left(\mathrm{CH}_{3} \mathrm{CH}=\mathrm{OH}\right)^{+}$and the comparatively large peak at $m / z 283$ 

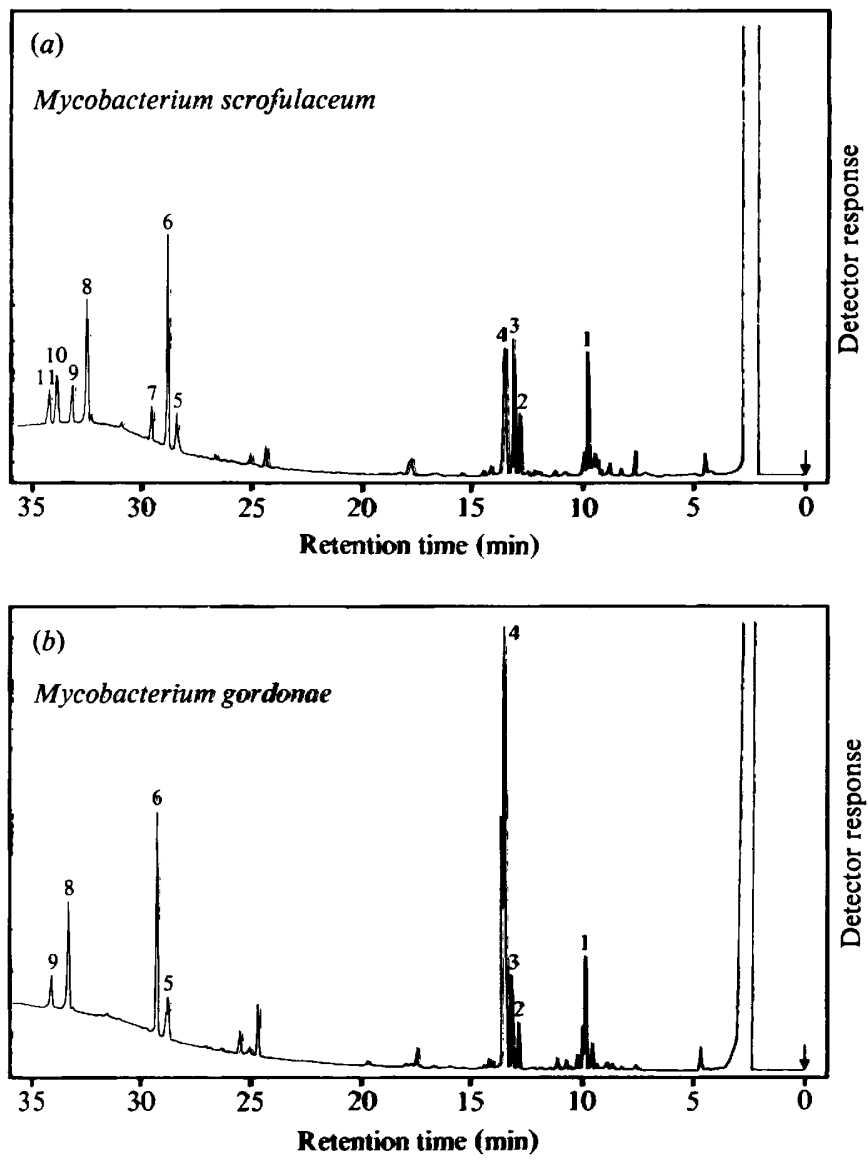

Fig. 1. Chromatograms obtained by analysis of one strain of (a) Mycobacterium scrofulaceum and (b) Mycobacterium gordonae. See text for specifications of analytical test conditions. Symbols: 1, TFA methyl-arabinoside; 2, TFA methyl-galactoside; 3, TFA methyl-mannoside; 4, TFA methyl-glucoside; 5 , methyl ester of $C_{16: 1}$ fatty acid; 6 , methyl ester of $C_{16: 0} ; 7$, TFA-2-octadecanol; 8, methyl ester of $\mathrm{C}_{\mathrm{t8:1}} ; 9$, methyl ester of $\mathrm{C}_{18: 0} ; 10$, TFA-2-eicosanol; 11 , methyl ester of $\mathrm{C}_{19: 0 \mathrm{Br}}$ (tuberculostearic acid).

$\left(\mathrm{CH}_{3}\left(\mathrm{CH}_{2}\right)_{17} \mathrm{CH}=\mathrm{OH}\right)^{+}$, tally with a proposed structure of 2-eicosanol for component (10). Analogously, the identity of peak (7) was established as 2-octadecanol.

\section{Chromatographic patterns}

The identities and relative areas of the peaks in the chromatograms of both of the species examined are given in Table 1.

Chromatograms representing $M$. gordonae exhibited a much larger peak of glucose than of other carbohydrates, e.g. galactose, arabinose and mannose. These chromatograms further differed from those of $M$. scrofulaceum by the absence of peaks representing 2-octadecanol, 2eicosanol and tuberculostearic acid, as registered by flame ionization detection. However, analysis of $M$. gordonae, using SIM revealed a peak having a retention time identical with that of the synthesized sample of methyl tuberculostearate, in an amount corresponding to less than $1 \%$ of that in M. scrofulaceum (Larsson et al., 1981).

\section{Reproducibility}

The standard deviations of the peak areas are given in Table 1. Chromatograms from studies on the duplicate cultures, and on the different strains of the same species, were similar, 
Table 1. Relative peak areas of chromatograms representing mycobacterial cellular constituents as produced by a flame ionization detector and an electronic integrator

Four strains of each species were used. The numbers in parentheses indicate the S.D.

Constituent
Arabinose
Galactose
Mannose
Glucose
$C_{16: 1}$
$C_{16: 0}$
2-Octadecanol
$C_{18: 1}$
$C_{18: 0}$
2-Eicosanol
$C_{19: 0 ~ B r}$

M. gordonae
$14.6(4 \cdot 4)$
$5.3(1 \cdot 0)$
$11.2(1.7)$
$68.8(6.6)$
$4.3(0.9)$
$47 \cdot 5(4 \cdot 4)$
0
$36.3(3.2)$
$9.4(1.4)$
0
0

$M$. scrofulaceum
$20.2(12 \cdot 4)$
$8.4(1 \cdot 8)$
$17.0(4 \cdot 2)$
$54.5(12 \cdot 9)$
$4.7(2 \cdot 0)$
$40 \cdot 1(3 \cdot 3)$
$5.0(2 \cdot 0)$
$18.8(4 \cdot 3)$
$18.3(5 \cdot 1)$
$10.4(1 \cdot 2)$
$6.8(0.8)$

especially with respect to the fatty acid patterns, while the carbohydrate peaks showed somewhat larger variations.

No differences could be observed between chromatograms representing preparations derivatized 1 week or 1 year after the mycobacteria had been lyophilized. Derivatized samples could be stored for at least 1 week at $-20^{\circ} \mathrm{C}$ without any change in their chromatographic patterns.

\section{DISCUSSION}

The analytic technique used in the present study, viz. acidic methanolysis of lyophilized bacterial cells followed by derivatization using trifluoroacetic anhydride prior to GC analysis, can be applied for characterization of bacteria in general, since all such organisms contain both carbohydrates and fatty acids. Alkaline saponification followed by esterification of cellular lipids has also been employed. This latter approach is rapid and easy to use, although not applicable for the study of both carbohydrates and fatty acids, since the former are degraded in the strongly alkaline environment employed during the saponification process.

The simultaneous determination by GC of bacterial carbohydrates and fatty acids can be achieved by using either trimethylsilyl or TFA derivatization of the methyl glycosides after acidic methanolysis (Jantzen et al., 1972). The latter derivatives are more volatile and heatstable than the former, but sensitive to moisture. Preferably, TFA derivatives should therefore be stored in an excess of reagent. The somewhat higher standard deviations of the peak areas of the TFA methyl glycosides than of the methyl esters found in the present study (Table 1) may be explained as resulting from incomplete TFA derivatization, or from degradation of the derivatives due to moisture in the atmosphere.

Larsson \& Mårdh (1976), who analysed the fatty acid and carbohydrate composition of some species of Mycobacterium using, in principle, the same test procedure as described in the present study, found reproducible differences between chromatograms of $\boldsymbol{M}$. avium, M. kansasii, $\boldsymbol{M}$. tuberculosis and $M$. bovis, strain BCG. The latter two species produced virtually identical fatty acid GC profiles, and could only be distinguished when carbohydrates were also analysed. The $2 \mathrm{~m}$ packed column used in this and also in a proceeding study (Larsson et al., 1979a), could scarcely separate the three hexoses. Furthermore, peaks of tuberculostearic acid and 2-eicosanol could not be separated. The capillary column in the present study, which used the same stationary phase (viz. OV-101) as the two previous studies, enabled all these compounds to be separated. This stationary phase possesses a high thermal stability and is consequently suitable for analysis of the long-chain fatty acids of mycobacteria.

In initial studies, it was found that the excess of TFA reagent in the samples had a marked deleterious effect on the capillary column. This was overcome by using the pre-column described, the packing material of which was regularly renewed. 
The finding of 2-octadecanol and 2-eicosanol in $\boldsymbol{M}$. scrofulaceum, but not in $\boldsymbol{M}$. gordonae, is of interest. The last-mentioned secondary alcohol has previously been found in the mycolic acids of a variety of mycobacterial species (Minnikin \& Goodfellow, 1980; Asselineau, 1966). The detection of these alcohols by using capillary columns can add to the differential diagnostic capability of the GC method used. In contrast to previous findings (Julak et al., 1980), we were able to detect tuberculostearic acid in $M$. gordonae by using SIM.

The GC technique described in the present paper appears to be useful for characterization of mycobacteria. Compared with the analysis of fatty acids alone, the method is more laborious and demanding, but elicits more information that might be of value in taxonomic studies on mycobacteria and possibly also on bacteria of other genera.

This study was supported by the Bengt Lundqvist Memorial Fund, the Swedish Association against Heart and Chest Diseases, and the Swedish Medical Research Council (grant no. 16X-05965).

\section{REFERENCES}

Asselineau, J. (1966). In The Bacterial Lipids, p. 69. Edited by E. Lederer. Paris: Hermann.

Guerrant, G. O., Lambert, M. A. \& Moss, C. W. (1981). Gas-chromatographic analysis of mycolic acid cleavage products in mycobacteria. Journal of Clinical Microbiology 13, 899-907.

JaNTzen, E., FrøHolm, L. O., HytTA, R. \& Bøvre, K. (1972). Gas chromatography of bacterial whole cell methanolysates. I. The usefulness of trimethylsilyl and trifluoroacetyl derivatives for strain and species characterization. Acta pathologica immunologica et microbiologica scandinavica B80, 660-671.

Julak, J., TuReCEK, F. \& Mikova, Z. (1980). Identification of characteristic branched-chain fatty acid of Mycobacterium kansasii and gordonae by gas chromatography-mass spectrometry. Journal of Chromatography 190, 183-187.

LARSSON, L. \& MÅRDH, P.-A. (1976). Gas chromatographic characterization of mycobacteria: analysis of fatty acids and trifluoroacetylated whole-cell methanolysates. Journal of Clinical Microbiology 3, 81-85.

LARSSON, L. \& MÅRDH, P.-A. (1977). Application of gas chromatography to diagnosis of microorganisms and infectious diseases. Acta pathologica immunologica et microbiologica scandinavica (supplement) 259, 5-15.

LARSSON, L., BERgMAN, R. \& MÅRDH, P.-A. (1979a). Gas chromatographic characterization of porcine and human strains belonging to the Mycobacterium avium-intracellulare complex. Acta pathologica immunologica et microbiologica scandinavica B87, 205-209.

LaRsSON, L., MARdH, P.-A. \& ODHAM, G. (1979b). Detection of tuberculostearic acid in mycobacteria and nocardiae by gas chromatography and mass spectrometry using selected ion monitoring. Journal of Chromatography, Biomedical Applications 163, 221223.

Larsson, L., MÅrdh, P.-A., OdhaM, G. \& WesterDAHL, G. (1980). Detection of tuberculostearic acid in biological specimens by means of glass capillary gas chromatography - electron and chemical ionization mass spectrometry, utilizing selected ion monitoring. Journal of Chromatography, Biomedical Applications 182, 402-408.

Larsson, L., MÅrdh, P.-A., Odham, G. \& WesterDAHL, G. (1981). Use of selected ion monitoring for detection of tuberculostearic and $C_{32}$ mycocerosic acid in mycobacteria and in five-day-old cultures of sputum specimens from patients with pulmonary tuberculosis. Acta pathologica immunologica et microbiologica scandinavica $\mathbf{B 8 9}, 245-251$.

MinNikin, D. E. \& Goodfellow, M. (1980). Lipid composition in the classification and identification of acid-fast bacteria. In Microbiological Classification and Identification, pp. 189-256. Edited by $\mathrm{M}$. Goodfellow \& R. G. Board. London: Academic Press.

Odham, G., Larsson, L. \& Mårdh, P.-A. (1979). Demonstration of tuberculostearic acid in sputum from patients with pulmonary tuberculosis by selected ion monitoring. Journal of Clinical Investigation 63, 813-819.

Tisdall, P. A., Roberts, G. O. \& Anhalt, J. P. (1979). Identification of clinical isolates of mycobacteria with gas-liquid chromatography alone. Journal of Clinical Microbiology 10, 506-514. 